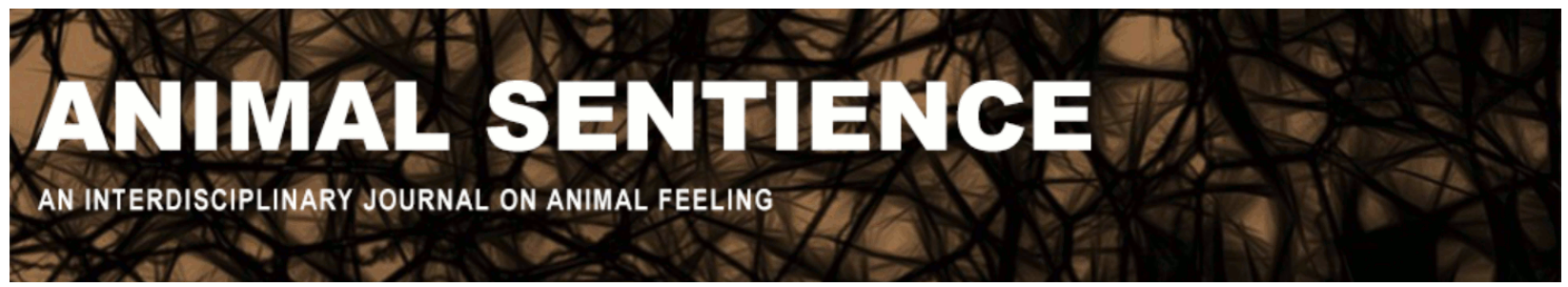

Seth, Anil K. (2016) Why fish pain cannot and should not be ruled out. Animal Sentience 3(14)

DOI: $10.51291 / 2377-7478.1038$

Date of submission: $2015-10-08$

Date of acceptance: 2015-12-13

(c)

This article has appeared in the journal Animal

Sentience, a peer-reviewed journal on animal

cognition and feeling. It has been made open access,

free for all, by WellBeing International and deposited

in the WBI Studies Repository. For more information,

please contact

wbisr-info@wellbeingintl.org.

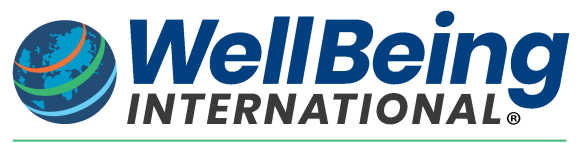

SOLUTIONS FOR PEOPLE, ANIMALS AND ENVIRONMENT 


\title{
Why fish pain cannot and should not be ruled out \\ Commentary on Key on Fish Pain
}

\author{
Anil K. Seth \\ School of Engineering and Informatics \\ University of Sussex
}

\begin{abstract}
Do fish consciously feel pain? Addressing this question, Key (2016) asks whether the neural mechanisms underlying conscious pain reports in humans can be identified in fish. This strategy fails in three ways. First, non-mammalian consciousness - if it exists - may depend on different mechanisms. Second, accumulating neurophysiological and behavioural evidence, evolutionary considerations, and emerging Bayesian brain theories suggest that if fish can feel at all, they can feel pain. Finally, the qualitative nature of pain and suffering obliges us, via the precautionary principle, to accommodate the possibility of its existence where doubt remains.
\end{abstract}

\begin{abstract}
Anil K. Seth a.k.seth@sussex.ac.uk is Professor of Cognitive and Computational Neuroscience and CoDirector of the Sackler Centre for Consciousness Science at the University of Sussex. www.anilseth.com
\end{abstract}

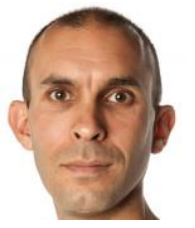

Key (2016) argues that fish do not consciously experience pain, on the general basis that brain mechanisms supporting conscious pain reports in humans cannot be identified in fish. It is refreshing to see a discussion of non-mammalian consciousness well informed by relevant (although mostly human) neurobiology. However, Key goes too far in making the strong inference against the existence of fish pain, for several reasons: (i) the biophysical substrates of conscious states (including pain and suffering) in any species, including humans, cannot yet be confidently identified; (ii) uncertainty about the mechanisms of human consciousness complicates the evaluation of potentially homologous processes in other species; and (iii) given this uncertainty, the qualitative nature of pain and suffering imposes moral and ethical imperatives (i.e., the precautionary principle) to be accommodating towards the possibility of its existence. There are also more positive reasons to consider that fish pain exists which fit comfortably with emerging Bayesian brain theories that emphasize active regulation of physiological homeostasis (Seth, 2015).

Key's argument can be summarized as follows. In humans, pain reports, indicating conscious pain, causally depend on specific cortical regions. This causal dependence, empirically supported by various brain lesion, imaging, and stimulation data, arises in virtue of specific structural and functional indicators of conscious processing within these regions. On a functional level, these include signal amplification and global integration across time and space. The structural properties proposed to support these functional indicators include parcellation of neural tissue into distinct (pain-related) regions; widespread recurrent connectivity among these regions, supported by discrete regional laminar and columnar organization; and topographical coding of pain-related information. Fish, the argument goes, 
lack plausible homologs of these properties and so the best interpretation of the current state of affairs is that fish do not consciously feel pain.

While this argument might seem to be about pain specifically, it is actually about consciousness generally. If fish lack the ability to consciously feel pain, it is (according to Key) because they lack the neuronal mechanisms needed to consciously experience (i.e., feel) anything at all, where these mechanisms are specified by the various structural and functional properties just mentioned. Thus Key's real claim, as I read it, is that fish do not consciously experience pain because they are wholly unconscious organisms sustained and guided by a highly adapted suite of reflex (including nociceptive) responses: Key's fish are philosophical zombie fish.

Can we be confident that the neuronal criteria identified by Key are necessary for conscious states? On one reading, Key seems to suggest that having a mammalian-like neocortex is necessary for consciousness. This strong position is easily challenged by a wealth of evidence from non-mammalian species like birds and cephalopods which display complex cognition and behaviour consistent with consciousness (D. B. Edelman \& Seth, 2009).

A more interesting interpretation is that the neocortex is important for mammalian consciousness in virtue of supporting specific functional properties like signal amplification ("ignition") and global integration. In human studies, much evidence supports the involvement of these properties in reportable conscious perception (Boly et al., 2013; Dehaene \& Changeux, 2011). Here it is important to distinguish between those mechanisms underlying explicit report from those that underlie the generation of the conscious scene itself (Aru, Bachmann, Singer, \& Melloni, 2012; de Graaf, Hsieh, \& Sack, 2012). Indeed, there has been recent excitement about "no report" paradigms, where the frontal cortical activations typically associated with conscious percepts (in humans) are no longer observed when participants are not required to report their conscious states (Frassle, Sommer, Jansen, Naber, \& Einhauser, 2014; see also Brascamp, Blake, \& Knapen, 2015, for a similar story regarding parietal activation ). Clearly, it would be a mistake to deny other species conscious states on the basis that they lack the neural machinery to report these states, independently of their ability to experience them (Seth, Baars, \& Edelman, 2005). And it is entirely possible that, should global integration and amplification turn out to be necessary for consciousness, these properties could be supported by alternative neuronal architectures in nonmammalian species (D. B. Edelman \& Seth, 2009).

Other criteria mentioned by Key are less readily associated with conscious report specifically. Strong local recurrence, perhaps dependent on specific laminar and columnar organization and bidirectional signal flow (Bastos et al., 2015), does seem consistently to be associated with conscious perception (Lamme, 2010) but is also a rather generic property of cortical and thalamocortical anatomy (Markov et al., 2013; Murphy, Duckett, \& Sillito, 1999). Other prominent theories of consciousness like "integrated information theory" (Tononi, 2008) associate consciousness with information-theoretic quantities which in principle can be realized by highly generic physical systems, including even non-biological artifacts (Tononi \& Koch, 2015). 
Might fish pain then depend on a different biophysical realization than human pain? On the face of it, this seems reasonable since conscious fish pain (if it exists) is likely to be qualitatively different from, and less differentiated than, human pain and suffering. Key excludes this possibility by saying that conscious fish pain of any kind would require the minimal neuronal architecture that he has already excluded from fish, where this minimal neuronal architecture is required for having conscious states of any kind. This again returns the argument to the basic, and unsolved, question of the neural substrates of consciousness in general, and away from a specific focus on pain.

Are there any positive reasons why fish might feel pain or have conscious experiences? Here it is useful to return to an evolutionary perspective on the functions of consciousness and of pain perception. While the function(s) of consciousness remain unestablished (just like its necessary and sufficient neural mechanisms), one plausible candidate is that conscious scenes integrate a broad range of organism-relevant signals in the service of flexible and adaptive behaviour (G. M. Edelman, 2003; Seth, 2009). As fish studies progress, it is apparent that many fish species have surprisingly sophisticated cognitive and behavioural repertoires, including long-term memory, social learning and cooperation, and even tool use (Brown, 2015). But the specific experience of pain speaks to something much more basic, which is the preservation of the integrity of the organism when under threat. In this light the ability to consciously feel pain would support the adaptive coordination of flexible responses to mitigate threats to physiological homeostasis, beyond that which can be supported by automatic nociceptive reflexes. Key might point out that fish sometimes do not display flexible responses to noxious stimuli, for instance in not showing obvious behaviour suppression following invasive craniotomy. Against this, other evidence suggests that fish show attention-related cognitive changes, for example, losing a normal fear of novel objects, following injection with acetic acid (Sneddon, 2003; see also Brown, 2015). In any case one might expect considerable cross-species variation in responses to pain (Reilly, Quinn, Cossins, \& Sneddon, 2008).

The active preservation of homeostatic integrity is a highly conserved evolutionary imperative which provides a rationale for the existence of basic conscious states characterized by affective qualia including pain (Panksepp, 2005). In humans, these affective conscious states leverage phylogenetically ancient brain regions and neurotransmitter systems which have more plausible homologs in non-mammalian species (including some fish) than the more recent neocortical extensions associated with "higher order" aspects of human consciousness (Damasio \& Carvalho, 2013; Demski, 2013; D. B. Edelman \& Seth, 2009). More speculatively, recent Bayesian theories of interoception and autonomic regulation (Seth, 2013, 2015) suggest that active regulation of physiological homeostasis may instantiate basic experiences of embodied selfhood which extend beyond modalityspecific experiences, and which in doing so may mediate a transition from pain to subjective suffering.

Do fish consciously feel pain? On the one hand, the lack of readily identifiable homologs of neural mechanisms associated with mammalian consciousness means we cannot assert definitely that fish are conscious. On the other, as we understand more about the mechanisms underlying human (and mammalian) consciousness, we will be better at assessing whether alternative realizations in other more distant species can do the same job. 
Other considerations, including those concerning active preservation of physiological integrity, provide positive reasons to believe in the possibility of fish consciousness. Should we then give fish "the 'benefit of the doubt' and unconditionally bestow them with the ability to feel pain?" as Key asks rhetorically. The right response is to say Yes to the first part, and No to the (hyperbolic) second part. Our current state of ignorance about consciousness together with the precautionary principle obliges us to take seriously that fish may feel pain or may suffer. And we are equally obliged to continue our efforts to understand the general neural mechanisms of consciousness, to dissolve our ignorance, so that crucial questions like those raised by Key can be resolved in a principled way.

\section{Acknowledgements}

A.K.S. is grateful to the Dr. Mortimer and Theresa Sackler Foundation, which supports the Sackler Centre for Consciousness Science.

\section{References}

Aru, J., Bachmann, T., Singer, W., \& Melloni, L. (2012). Distilling the neural correlates of consciousness. Neuroscience and biobehavioral reviews, 36(2), 737-746. doi: 10.1016/j.neubiorev.2011.12.003

Bastos, A. M., Vezoli, J., Bosman, C. A., Schoffelen, J. M., Oostenveld, R., Dowdall, J. R., De Weerd, P., Kennedy, H., \& Fries, P. (2015). Visual areas exert feedforward and feedback influences through distinct frequency channels. Neuron, 85(2), 390-401. doi: 10.1016/j.neuron.2014.12.018

Boly, M., Seth, A. K., Wilke, M., Ingmundson, P., Baars, B., Laureys, S., Edelman, D. B., \& Tsuchiya, N. (2013). Consciousness in humans and non-human animals: recent advances and future directions. Front Psychol, 4, 625. doi: 10.3389/fpsyg.2013.00625

Brascamp, J., Blake, R., \& Knapen, T. (2015). Negligible fronto-parietal BOLD activity accompanying unreportable switches in bistable perception. Nat Neurosci. doi: $10.1038 / \mathrm{nn} .4130$

Brown, C. (2015). Fish intelligence, sentience and ethics. Anim Cogn, 18(1), 1-17. doi: 10.1007/s10071-014-0761-0

Damasio, A., \& Carvalho, G. B. (2013). The nature of feelings: evolutionary and neurobiological origins. Nature reviews. Neuroscience, 14(2), 143-152. doi: $10.1038 / \mathrm{nrn} 3403$

De Graaf, T. A., Hsieh, P. J., \& Sack, A. T. (2012). The 'correlates' in neural correlates of consciousness. Neurosci Biobehav Rev, 36(1), 191-197. doi:

10.1016/j.neubiorev.2011.05.012 
Dehaene, S., \& Changeux, J. P. (2011). Experimental and theoretical approaches to conscious processing. Neuron, 70(2), 200-227. doi: S0896-6273(11)00258-3

[pii]10.1016/j.neuron.2011.03.018

Demski, L. S. (2013). The pallium and mind/behavior relationships in teleost fishes. Brain Behav Evol, 82(1), 31-44. doi: 10.1159/000351994

Edelman, D. B., \& Seth, A. K. (2009). Animal consciousness: a synthetic approach. Trends Neurosci, 32(9), 476-484.

Edelman, G. M. (2003). Naturalizing consciousness: a theoretical framework. Proc Natl Acad Sci U S A, 100(9), 5520-5524.

Frassle, S., Sommer, J., Jansen, A., Naber, M., \& Einhauser, W. (2014). Binocular rivalry: frontal activity relates to introspection and action but not to perception. $J$ Neurosci, 34(5), 1738-1747. doi: 10.1523/JNEUROSCI.4403-13.2014

Key, B. (2016). Why fish do not feel pain. Animal Sentience 2016.003.

Lamme, V. A. (2010). How neuroscience will change our view on consciousness. Cognitive Neuroscience, 1(3), 204-240.

Markov, N. T., Ercsey-Ravasz, M., Van Essen, D. C., Knoblauch, K., Toroczkai, Z., \& Kennedy, H. (2013). Cortical high-density counterstream architectures. Science, 342(6158), 1238406. doi: 10.1126/science.1238406

Murphy, P. C., Duckett, S. G., \& Sillito, A. M. (1999). Feedback connections to the lateral geniculate nucleus and cortical response properties. Science, 286(5444), 1552-1554.

Panksepp, J. (2005). Affective consciousness: Core emotional feelings in animals and humans. Conscious Cogn, 14(1), 30-80.

Reilly, S.C., Quinn, J.P., Cossins, A.R., \& Sneddon, L. U. (2008). Behavioural analysis of a nociceptive event in fish: comparisons between three species demonstrate specific responses. Appl Anim Behav Sci Am, 114, 248-259.

Seth, A. K. (2009). Functions of consciousness. In W. P. Banks (Ed.), Elsevier Encyclopedia of Consciousness: Elsevier.

Seth, A. K. (2013). Interoceptive inference, emotion, and the embodied self. Trends Cogn Sci, 17(11), 565-573. doi: 10.1016/j.tics.2013.09.007

Seth, A. K. (2015). The Cybernetic Bayesian Brain. In T. Metzinger \& J. M. Windt (Eds.), Open MIND (pp. 1-24). Frankfurt, Germany: MIND Group.

Seth, A. K., Baars, B. J., \& Edelman, D.B. (2005). Criteria for consciousness in humans and other mammals. Consciousness and Cognition, 14(1), 119-139. 
Sneddon, L. U. (2003). The evidence for pain in fish: the use of morphine as an analgesic. Appl Anim Behav Sci, 83, 153-162.

Tononi, G. (2008). Consciousness as integrated information: a provisional manifesto. Biol Bull, 215(3), 216-242.

Tononi, G., \& Koch, C. (2015). Consciousness: here, there and everywhere? Philos Trans $R$ Soc Lond B Biol Sci, 370(1668). doi: 10.1098/rstb.2014.0167 